\title{
SwissDRG 6.0: aktualisierte Strategie in Zukunft notwendig
}

\section{Petra Ingenpass ${ }^{a}$, Beatrix Meyer}

${ }^{a}$ Dr. med., Stv. Leiterin Abteilung Tarife und Gesundheitsökonomie Spitalärzte; ${ }^{b}$ Leiterin Abteilung Tarife und Gesundheitsökonomie Spitalärzte

Für die SwissDRG-Version 6.0 erfolgten wiederum zahlreiche Umbauten bei immer noch ungenügender Datenqualität. Der Zeitpunkt für eine Standortbestimmung mit Aktualisierung der Entwicklungsstrategie ist gekommen.

\section{Aktuelle Strategie und Massnahmen zu deren Umsetzung festlegen}

Die Weiterentwicklung von SwissDRG basiert auf der Entwicklungsstrategie 2013+, welche die SwissDRG AG 2012 mit ihren Partnern erarbeitete. Während einige strategische Ziele bereits erreicht wurden, zeichnen sich bei anderen nur geringe Fortschritte ab. Der Zeitpunkt für eine Analyse des aktuellen Entwicklungsstands von SwissDRG ist deshalb aus Sicht der FMH gekommen. Anhand dieser Analyse sind Schlussfolgerungen für die künftig einzuschlagende Entwicklungsrichtung zu ziehen. Die FMH erachtet es als wichtig, dass die SwissDRG AG eine aktualisierte Strategie erarbeitet und aufzeigt, mit welchen Massnahmen diese umgesetzt werden kann.

Gemäss der Entwicklungsstrategie 2013+ der SwissDRG AG soll die Tarifstruktur anhand von rund 1200 DRGs einschliesslich Zusatzentgelten die Kostenunterschiede zwischen den Leistungserbringern bestmöglich erklären. Fraglich ist, inwiefern diese Vorgabe beispielsweise mit der vom Verwaltungsrat der SwissDRG AG aktuell festgelegten weiteren Ausdifferenzierung der Tarifstruktur kompatibel ist, um sehr teure und hochdefizitäre Fälle besser abzubilden. Eine Zunahme an Fallpauschalen ist dabei wahrscheinlich. Auch sind weitere Zusatzentgelte zu erwarten und notwendig tragen sie doch zu einer sachgerechteren Vergütung bei. Das ist eines von vielen Beispielen, weshalb es eine Überprüfung der bestehenden Strategie braucht.

\section{Tarifstruktur verbessern - Verwaltungs- rat gibt Richtwert vor}

Gemäss der SwissDRG AG ist davon auszugehen, dass Fallpauschalen nicht alle Kostenunterschiede zwischen den Spitälern erklären. So bestehen regionale Unterschiede bei den Lohn- und Lohnnebenkosten. Zu berücksichtigen sind zudem Vorhalteleistungen für hoch- spezialisierte Infrastrukturen oder Notfallversorgung, die notwendig, aber nicht voll ausgelastet sind (z.B. Sonderisolierstationen). Aber auch nicht durch Gewinnfälle kompensierte Hochdefizitfälle verursachen Verluste. Solche Kostenunterschiede, die nicht auf eine unterschiedliche Effizienz der Spitäler zurückzuführen sind, können in den Tarifverhandlungen mittels Preisdifferenzierung aufgefangen werden.

Der Verwaltungsrat der SwissDRG AG sieht bei korrekter Ausscheidung der OKP-relevanten Betriebs- und Anlagenutzungskosten einen Richtwert für einen Korridor der kalkulatorischen Durchschnittsfallkosten von $\pm 5 \%$ vor; dieses Ziel soll dabei bis Ende 2019 erreicht werden. Aus Sicht der FMH ist es zentral, dass die SwissDRG AG in ihrer Strategie aufzeigt, mit welchen Massnahmen dieses Ziel erreicht wird bzw. wie die Tarifstruktur entsprechend verbessert werden kann.

\section{Detaillierter Abschlussbericht für mehr Transparenz gefordert}

In der Präsentation der SwissDRG-Version 6.0 geht die SwissDRG AG auf mehrere Neuerungen und Umbauten ein. Der Detaillierungsgrad und die Relevanz dieser Informationen sind dabei zum Teil sehr unterschiedlich. So wird die Zusammenführung aller Leistungen bei Appendizitis zur besseren Übersicht ausführlich dargestellt, während auf die mit der Version 6.0 neu vorgenommene Bewertung bisher unbewerteter DRGs der Frührehabilitation nicht vertieft eingegangen wird. In der Version 6.0 wurden verschiedene Leistungen in die Prä-MDC ${ }^{1}$ verschoben, in welcher ursprünglich teure, aber auch fachübergreifende Behandlungen wie z.B. Transplantationen oder Beatmungen abgebildet waren. Gemeinsame Merkmale der aus den Organkapiteln in die Prä-MDC verschobenen DRGs können dabei eine Komplexbehandlung, Intermediate Care oder eine Frührehabilitation sein. Hintergründe zu diesem Vorgehen finden sich kaum. Dadurch können zwar DRG-Splits oder das Ansteuern von Fehler-DRGs vermieden werden, es bedeutet aber auch, dass Prozeduren immer mehr Gewicht beigemessen wird und Diagnosen hingegen an Bedeutung einbüssen. Die 
FMH erachtet es als notwendig, dass die SwissDRG AG Entwicklungen und Änderungen in der Tarifstruktur in einem ausführlichen und detaillierten Abschlussbericht transparent und nachvollziehbar darstellt.

\section{Verbesserung der Datenlage - ein Dauerbrenner}

Zur Verbesserung der Datenqualität hat die SwissDRG AG bereits verschiedene Massnahmen eingeleitet, beispielsweise die Pflicht zur Erhebung der Fallkosten nach REKOLE $^{\circledR}$. Weiter hat sie ihren Austausch mit den Spitälern für die Datenplausibilisierung intensiviert und bietet ihnen nun auch die Möglichkeit unterjähriger Plausibilisierungen über ein Datenportal an. Gemäss der SwissDRG AG besteht jedoch weiterhin ein deutlicher Verbesserungsbedarf, sowohl bei der Erhebung der Kostendaten als auch bei der Kodierung erbrachter Leistungen. So mussten für die Entwicklung von SwissDRG 6.0 die Gesamtdaten von 16 Spitälern gelöscht werden.

Die Datenqualität der Anlagenutzungskosten (ANK) kann die SwissDRG AG derzeit nicht einschätzen. ${ }^{2}$ Sie dürfte sich aber kaum verbessert haben; so ist die Spannbreite des mittleren ANK-Anteils an den Gesamtkosten zwischen den Spitälern mit rund $4 \%$ bis $18 \%$ nach wie vor sehr hoch. 2015 hat die SwissDRG AG den Beschluss gefasst, dass Spitäler die ANK ab dem Datenjahr 2016 zur Weiterentwicklung von SwissDRG ausschliesslich nach REKOLE $^{\circledR}$ liefern müssen. Diese Massnahme ist zwar wichtig; fraglich ist jedoch, ob sie ausreicht, um die erforderliche Qualität der ANK-Daten zu erreichen.

Der Anspruch auf eine Verbesserung der Datenqualität fand bereits Eingang in die Entwicklungsstrategie 2013+. Da die bisherigen Massnahmen zur Erzielung einer genügenden Datenqualität nicht ausreichten, sind weitere zu prüfen. Gleichzeitig ist in den strategischen Zielen die Verfahrensweise zu definieren, sofern sich keine nachhaltige Verbesserung bei der Datenqualität einstellt.

\section{Problematik der Hochdefizitfälle zügig an die Hand nehmen}

Die detaillierte Analyse zur SwissDRG-Version 6.0 finden Sie in der FMH-Stellungnahme: www.fmh.ch $\rightarrow$ Stationäre Tarife $\rightarrow$ Positionen $\rightarrow$ Stellung nahmen.

Korrespondenz: FMH Frohburgstrasse 15 CH-4600 Olten Tel. 0313591111 Fax 0313591112 tarife.spital[at]fmh.ch
AG Auswertungen zu teuren und hochdefizitären Fällen. Dabei legt sie die Definition des Hochdefizitfalles mit Gesamtfallkosten von 400000 Franken und einem Defizit von 40000 Franken zugrunde. Diese Definition erfüllen bei der SwissDRG Version 6.0 insgesamt 76 Fälle aus 14 Spitälern, die damit total ein Defizitvolumen von 12 Mio. Franken generieren. Für die Version 5.0 waren es 56 Fälle aus 12 Spitälern mit einem Defizitvolumen von 11 Mio. Franken. ${ }^{3}$ Aus Sicht der FMH ist der Einbezug der Gesamtkosten in die Definition des Hochdefizitfalles ungeeignet - relevant ist nur die Höhe des Defizits.

Um hochdefizitäre Behandlungsfälle besser abzubilden, sind alle im gesteckten Rahmen möglichen Lösungsansätze zu verfolgen. Die SwissDRG AG überprüfte dazu beispielsweise erneut die Zuschläge für Langlieger. Für 347 Fallpauschalen wurde der DRG-individuelle High Outlier-Zuschlag angepasst. Es gilt aber auch, entsprechende Entwicklungen des G-DRG-Systems auf Kompatibilität hin zu prüfen, wie beispielsweise eine Erweiterung des Komplikations- und Komorbiditäts-Levels.

\section{Frühreha-DRGs allesamt bewertet - wenn auch auf nicht robuster Datenbasis}

Mit der SwissDRG Version 6.0 sind erstmals alle Frührehabilitations-DRGs bewertet. Damit wurde die Forderung des Bundesrates erfüllt, nicht bewertete Fallpauschalen möglichst zu vermeiden. Neurologischneurochirurgische und fachübergreifende FrührehaFälle werden entweder zwei Hauptdiagnose-unabhängigen DRGs der Prä-MDC oder zwei DRGs der Neurologie zugeordnet. Während die neurologisch-neurochirurgische Frührehabilitation klar definiert ist, fehlt für die fachübergreifende Frührehabilitation die Festlegung von Mindestmerkmalen. Der Code muss dringend unter Einbezug aller betroffenen Fachgesellschaften definiert werden.

\section{Fazit der FMH}

Der Zeitpunkt für eine Standortbestimmung ist gekommen. Aus Sicht der FMH ist es wichtig, dass die SwissDRG AG eine aktualisierte Strategie erarbeitet und aufzeigt, mit welchen Massnahmen die strategischen Ziele Schritt um Schritt umgesetzt werden können. Insbesondere gilt es darzulegen, wie die Tarifstruktur verbessert und der vom Verwaltungsrat vorgegebene Richtwert für einen Korridor der kalkulatorischen Durchschnittsfallkosten von $\pm 5 \%$ bis Ende 2019 erreicht werden kann Schliesslich geht es darum, mehr Transparenz in die Entwicklungen der Tarifstruktur zu bringen. Auch für die kommenden Jahre müssen also noch einige Herausforderungen gemeistert werden. 